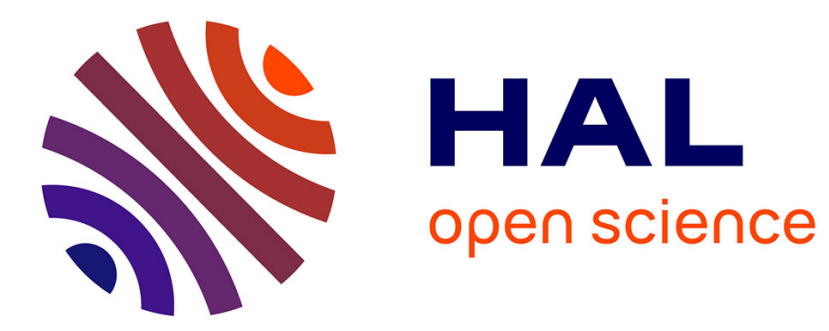

\title{
Bandwidth assignment for reliable fixed broadband wireless networks
}

Grit Classen, David Coudert, Arie Koster, Napoleao Nepomuceno

\section{To cite this version:}

Grit Classen, David Coudert, Arie Koster, Napoleao Nepomuceno. Bandwidth assignment for reliable fixed broadband wireless networks. 12th IEEE International Symposium on a World of Wireless, Mobile and Multimedia Networks (WoWMoM), Jun 2011, Lucca, Italy. pp.1-6, 10.1109/WoWMoM.2011.5986471 . inria-00587698

\section{HAL Id: inria-00587698 https://hal.inria.fr/inria-00587698}

Submitted on 21 Apr 2011

HAL is a multi-disciplinary open access archive for the deposit and dissemination of scientific research documents, whether they are published or not. The documents may come from teaching and research institutions in France or abroad, or from public or private research centers.
L'archive ouverte pluridisciplinaire HAL, est destinée au dépôt et à la diffusion de documents scientifiques de niveau recherche, publiés ou non, émanant des établissements d'enseignement et de recherche français ou étrangers, des laboratoires publics ou privés. 


\title{
Bandwidth assignment for reliable fixed broadband wireless networks
}

\author{
Grit Claßen \\ Lehrstuhl II für Mathematik \\ RWTH Aachen University, 52056 Aachen, Germany \\ classen@math2.rwth-aachen.de \\ David Coudert \\ Mascotte, INRIA, I3S, CNRS, Université de Nice Sophia \\ Sophia Antipolis, France \\ david.coudert@inria.fr \\ Arie M. C. A. Koster \\ Lehrstuhl II für Mathematik \\ RWTH Aachen University, 52056 Aachen, Germany \\ koster@math2.rwth-aachen.de \\ Napoleão Nepomuceno \\ Institut for Matematik og Datalogi \\ Syddansk Universitet, Campusvej 55, DK-5230 Odense \\ napoleao@imada.sdu.dk
}

\begin{abstract}
In this paper, we investigate on conceiving reliable fixed broadband wireless networks under outage probability constraints. We introduce a joint model of data routing and bandwidth assignment that minimizes the total renewal fees of licenses. This problem differs from classical capacity planning since the capacity of microwave links is prone to variations and, hence, we must deal with random parameters to guarantee a desirable reliability level of the solution. We introduce a chance-constrained programming approach to tackle this problem and derive integer linear programming (ILP) counterparts. We further propose cutset-based valid inequalities to enhance the performance of ILP solvers. Computational results illustrate the price of reliability and present a comparative study on the performance of the different formulations.
\end{abstract}

\section{Introduction}

Fixed broadband wireless communications is a particular sector of the communication industry that holds great promise for delivering private high-speed data connections by means of microwave radio transmission [1, 7]. Microwave, in the context of this work, refers to terrestrial point-to-point digital radio communications, usually employing highly directional antennas in clear line-of-sight (LOS) and operating in licensed frequency bands. This makes microwave communications typically free from interference. 
Despite recent advances in fixed broadband wireless communications, a variety of questions remain unaddressed in this area. Particularly, capacity planning in fixed wireless networks is quite different from wired network planning. In fact, the radio frequency spectrum is a limited natural resource which has been regulated worldwide to promote its efficient use. Moreover, environment conditions (e.g., weather) play an important role since they can introduce instantaneous variations into the communication channel, likely leading to outage events.

Although having limited bandwidth and suffering channel impairments, fixed wireless networks must degrade smoothly as environment conditions degrade. As a common practice, operators highly overprovision bandwidth during network planning to avoid traffic bottlenecks under adverse scenarios (when the performance of some links deteriorates). This approach, however, incurs additional investments that do not result in resource- and cost-efficient networks, besides leading to an inefficient use of the radio spectrum.

In this paper, we introduce a chance-constrained mathematical programming approach to conceive reliable fixed broadband wireless networks under outage probability constraints. Chanceconstrained programming is a specific model of stochastic optimization for dealing with random parameters in optimization problems [9, 11]. Actually, there exist situations where constraint violation can hardly be avoided because of unexpected extreme events. This approach thus aims at determining optimal decisions that have to be taken prior to the observation of random parameters and remain feasible for a given infeasibility tolerance.

Chance-constrained programming is still considered as hard and widely intractable since the feasible region defined by a probabilistic constraint is generally not convex. In addition, among the vast literature on chance-constrained programming, few research work has been carried out to tackle combinatorial problems [5, 8. Given these difficulties, we derive an equivalent ILP formulation for the case where the outage probabilities of the microwave links are independent and propose cutsetbased valid inequalities to obtain strengthened formulations for this problem.

The remainder of the paper is organized as follows. In Section 2, we briefly discuss spectrum pricing in licensed bands and convey information about the link characterization. In Section 3 , we introduce exact formulations for the application considered here. Section 4 is devoted to cutsetbased valid inequalities. In Section 5 , we illustrate the price of reliability and present a comparative study of the different formulations. Final remarks and comments on future work conclude the paper with Section 6 .

\section{Preliminaries}

\subsection{Licensed frequency bands}

The radio frequency spectrum is a limited natural resource regulated worldwide by the International Telecommunications Union (ITU). In conjunction with ITU regulations, national legislation instruments establish the availability of frequency bands for specific applications and the procedures for issuing licenses. A license (assignment) is the authorization given by an administration for a radio station to use a radio frequency under specified conditions, normally subject to renewal upon payment of renewal fees.

Administrative methods of setting spectrum prices are increasingly being supplemented by the use of market-based methods. In some countries, the frequency spectrum is sold to an operator, either by auction or by competitive tender. In this case, once an operator is assigned the privilege 


\begin{tabular}{lllcc}
\hline $\begin{array}{l}\text { Modulation } \\
\text { scheme }\end{array}$ & $\begin{array}{l}\text { Bandwidth } \\
\text { efficiency }\end{array}$ & $\begin{array}{l}\text { SNR } \\
\text { requirem. }\end{array}$ & $\begin{array}{l}\text { Capacity } \\
\text { for } 7 \mathrm{MHz}\end{array}$ & $\begin{array}{l}\text { Capacity } \\
\text { for } 28 \mathrm{MHz}\end{array}$ \\
\hline QPSK & $2 \mathrm{bps} / \mathrm{Hz}$ & $14.21 \mathrm{~dB}$ & $14 \mathrm{Mbps}$ & $56 \mathrm{Mbps}$ \\
16-QAM & $4 \mathrm{bps} / \mathrm{Hz}$ & $21.02 \mathrm{~dB}$ & $28 \mathrm{Mbps}$ & $112 \mathrm{Mbps}$ \\
32-QAM & $5 \mathrm{bps} / \mathrm{Hz}$ & $25.24 \mathrm{~dB}$ & $35 \mathrm{Mbps}$ & $140 \mathrm{Mbps}$ \\
64-QAM & $6 \mathrm{bps} / \mathrm{Hz}$ & $27.45 \mathrm{~dB}$ & $42 \mathrm{Mbps}$ & $168 \mathrm{Mbps}$ \\
128-QAM & $7 \mathrm{bps} / \mathrm{Hz}$ & $31.10 \mathrm{~dB}$ & $49 \mathrm{Mbps}$ & $196 \mathrm{Mbps}$ \\
256-QAM & $8 \mathrm{bps} / \mathrm{Hz}$ & $33.78 \mathrm{~dB}$ & $56 \mathrm{Mbps}$ & $224 \mathrm{Mbps}$ \\
\hline
\end{tabular}

Table 1: Bandwidth efficiency, SNR requirement, and capacity.

to use a part of the spectrum, the cost to the operator would be the same regardless whether or not a link transmits on certain bandwidth. However, in most cases (as assumed in this paper), the price of a frequency spectrum for a single microwave link is a function of the amount of spectrum (bandwidth) in $\mathrm{MHz}$ with which a license is associated.

\subsection{Link characterization}

Commonly, to support broadband applications, modern microwave systems use quadrature amplitude modulation (QAM). An $m$-QAM scheme presents $m$ combinations of amplitude and phase, each one representing an $n$-bit pattern called a symbol (with $n=\log _{2} m$ and integer). Given the channel bandwidth $B$ and the $m$-QAM scheme in use, we can approximate the channel capacity $C$ by:

$$
C[b p s]=n \cdot B[H z]
$$

High-level QAM schemes, despite presenting better bandwidth efficiency, are more susceptible to errors due to channel impairments. As the modulation scheme changes to accommodate higher data rates, the signal-to-noise ratio (SNR) requirement increases to preserve the bit error rate (BER) (see Table 1 [2]). Rigorously, we can also use different error correction codes. In any case, we can rebuild this table for different combinations of modulation and coding (and other radio parameters) based on equipment specifications.

Since the transmitted signal suffers deep fades, microwave links are susceptible to outage events. Fading phenomena are described in statistical terms, and the probability of fades of a particular magnitude can be evaluated through analytical techniques [12, 3]. To overcome outage events, modern microwave systems employ adaptive modulation and coding which has been proven to considerably enhance link performance [4]. To keep the BER performance, this technique entails the variability of the link's capacity.

Considering a finite set of efficient radio configurations (for which no configuration that presents better bandwidth efficiency for a lower SNR requirement exists), we can associate a discrete probability distribution with these configurations, obtained either from statistical studies (in case of license renewal of a network in operation) or from fading models and power budget calculations. We henceforth assume that such a discrete probability distribution is known for each microwave link and bandwidth. 


\section{Mathematical formulations}

In this section, we introduce chance-constrained mathematical formulations and their ILP counterparts to the optimization problem of deciding the bandwidth assignment and network flows that minimize the total bandwidth cost, while handling all the traffic requirements simultaneously with a given reliability level.

\subsection{Separate chance constraints}

The network's topology is modeled as a digraph $G=(V, E)$, where each node $v \in V$ denotes a radio base station (RBS) and each arc $u v \in E$ represents a microwave link from $u$ to $v$, with $u, v \in V$ and $u \neq v$. Let $\delta^{+}(v)\left(\delta^{-}(v)\right)$ denote the set of outneighbors (inneighbors) of $v$. Let $W_{u v}$ be the number of bandwidth choices available for arc $u v \in E$. Each bandwidth $b_{u v}^{w}$, for $w=1, \ldots, W_{u v}$, is associated with its $\operatorname{cost} c_{u v}^{w}$ and a random variable $\eta_{u v}^{w}$ that represents the bandwidth efficiency of the current radio configuration. Let $\varepsilon_{u v}>0$ be the infeasibility tolerance (typically near zero) on link $u v$ chosen by the network engineer. The traffic requirements are defined by $K$ oriented pairs of nodes $\left(s^{k}, t^{k}\right)$, with $s^{k}, t^{k} \in V$ and $s^{k} \neq t^{k}$, and expected demand $d^{k}$ of pair $k=1, \ldots, K$.

We aim at determining the bandwidth assignment and the traffic flows that minimize the total bandwidth cost. Let $y_{u v}^{w}$ be the binary decision variable indicating whether the bandwidth $b_{u v}^{w}$, $w=1, \ldots, W_{u v}$, is assigned or not for arc $u v \in E$. The flow variables $f_{u v}^{k}$ denote the fraction of $d^{k}$, $k=1, \ldots, K$, routed on arc $u v \in E$. The optimization problem can be formulated as follows:

$$
\begin{aligned}
& \min \sum_{u v \in E} \sum_{w=1}^{W_{u v}} c_{u v}^{w} y_{u v}^{w}
\end{aligned}
$$

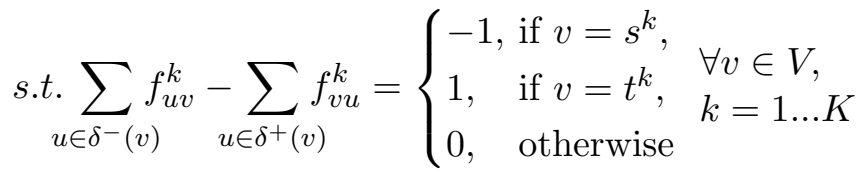

$$
\begin{aligned}
& \mathcal{P}\left(\sum_{k=1}^{K} d^{k} f_{u v}^{k} \leq \sum_{w=1}^{W_{u v}} \eta_{u v}^{w} b_{u v}^{w} y_{u v}^{w}\right) \geq 1-\varepsilon_{u v} \forall u v \in E \\
& \sum_{w=1}^{W_{u v}} y_{u v}^{w}=1 \quad \forall u v \in E \\
& f_{u v}^{k} \in[0,1], y_{u v}^{w} \in\{0,1\}
\end{aligned}
$$

The objective function (1) represents the total bandwidth cost that is to minimize. The flow conservation property is expressed by (2), guaranteeing that the traffic requirements are entirely fulfilled. Constraints (3) ensure that the available capacity on each link (considering the bandwidth choice and the random configuration) supports the total traffic to be routed through it with (high) probability $1-\varepsilon_{u v}$. Finally, the bandwidth selection is determined by (4).

Since we have a finite number of scenarios, this probabilistic program can be equivalently written as a standard ILP model. However, this model is highly intractable due to the very large number of scenarios to be considered. Here, we use the idea of basic scenarios [5] to obtain, in an efficient 
way, the deterministic counterparts of constraints (3):

$$
\sum_{k=1}^{K} d^{k} f_{u v}^{k} \leq \sum_{w=1}^{W_{u v}} n_{u v}^{w} b_{u v}^{w} y_{u v}^{w} \quad \forall u v \in E
$$

where, for each link and bandwidth, the constant $n_{u v}^{w}$ represents the maximum bandwidth efficiency we can assume taking into account the infeasibility tolerance $\varepsilon_{u v}$. It can be easily computed from the most bandwidth-efficient configuration for which the probability that the link is operated at this configuration or higher is at least $1-\varepsilon_{u v}$.

Since we impose separate probabilistic constraints (3) on each link, even if we consider a very small infeasibility tolerance on each constraint, the optimal solution can be infeasible with a significant probability when the number of links increases. Therefore, this approach is worthwhile for particular cases where the network is not too large and the links are engineered to have a very high availability. In the sequel, we present a joint chance-constrained program to overcome this limitation.

\subsection{Joint chance constraints}

We now enforce an infeasibility tolerance on the entire block of capacity constraints, guaranteeing that the assigned bandwidth supports the total traffic to be routed through the network with (high) probability $1-\varepsilon$. Thus, constraints (3) are now replaced by a single chance constraint:

$$
\mathcal{P}\left(\sum_{k=1}^{K} d^{k} f_{u v}^{k} \leq \sum_{w=1}^{W_{u v}} \eta_{u v}^{w} b_{u v}^{w} y_{u v}^{w} \quad \forall u v \in E\right) \geq 1-\varepsilon
$$

In case of independent probabilities, we can reformulate the left hand side of (7) as the product of probabilities. For this, we introduce the following modifications to the previous formulation: Let $M_{u v}^{w}$ be the number of configurations held by arc $u v$ with respect to the bandwidth choice $w$. Let

$\rho_{u v}^{w m}$ be the probability that the link is operated at configuration $m$ or higher. Now $b_{u v}^{w m}$ represents the capacity on arc $u v$ for a given bandwidth choice $w$ and a specific configuration $m$. In addition, the binary decision variables $y$ obtain a new index $m$ that incorporates the assumption on the radio configuration. The problem can be rewritten as: 


$$
\begin{aligned}
& \min \sum_{u v \in E} \sum_{w=1}^{W_{u v}} \sum_{m=1}^{M_{u v}^{w}} c_{u v}^{w} y_{u v}^{w m} \\
& \text { s.t. } \sum_{u \in \delta^{-}(v)} f_{u v}^{k}-\sum_{u \in \delta^{+}(v)} f_{v u}^{k}=\left\{\begin{array}{ll}
-1, & \text { if } v=s^{k}, \\
1, & \text { if } v=t^{k}, \quad \forall v \in V, \\
0, & \text { otherwise }
\end{array} \quad k=1 . . K\right. \\
& \sum_{k=1}^{K} d^{k} f_{u v}^{k} \leq \sum_{w=1}^{W_{u v}} \sum_{m=1}^{M_{u v}^{w}} b_{u v}^{w m} y_{u v}^{w m} \quad \forall u v \in E \\
& \prod_{u v \in E}\left(\sum_{w=1}^{W_{u v}} \sum_{m=1}^{M_{u v}^{w}} \rho_{u v}^{w m} y_{u v}^{w m}\right) \geq 1-\varepsilon \\
& \sum_{w=1}^{W_{u v}} \sum_{m=1}^{M_{u v}^{w}} y_{u v}^{w m}=1 \\
& f_{u v}^{k} \in[0,1], y_{u v}^{w m} \in\{0,1\}
\end{aligned}
$$

Note that now, in the capacity constraints (10), we assume explicitly a hypothesis on the radio configuration. Obviously, more conservative hypotheses lead to more reliable solutions. Constraint (11) denotes formally this relation. According to the bandwidth assignment and the hypotheses on the radio configuration, it guarantees that the confidence of the solutions is at least $1-\varepsilon$. Constraint (11) is not linear, but it can be easily linearized: By employing monotonicity of logarithmic functions and because the logarithm of a product is equal to the sum of the logarithms, (11) is equivalent to

$$
\sum_{u v \in E} \log \left(\sum_{w=1}^{W_{u v}} \sum_{m=1}^{M_{u v}^{w}} \rho_{u v}^{w m} y_{u v}^{w m}\right) \geq \log (1-\varepsilon)
$$

By (12), exactly one of the sum elements within the logarithmic function will be nonzero. Hence, (14) is equivalent to

$$
\sum_{u v \in E} \sum_{w=1}^{W_{u v}} \sum_{m=1}^{M_{u v}^{w}} \log \left(\rho_{u v}^{w m}\right) y_{u v}^{w m} \geq \log (1-\varepsilon)
$$

\section{$4 \quad$ Valid inequalities}

Constraints (9), (10), (12) define a classical network design problem studied intensively in the literature (see [10] and the references therein). Several valid inequalities have been introduced to this problem, in particular, so-called cut-based inequalities. Let $S \subset V$ be a proper and nonempty subset of $V$ and $\bar{S}=V \backslash S$ its complement. The set $(S, \bar{S}):=\{u v \in E: u \in S, v \in \bar{S}\}$ is a cutset. Let $K_{(S, \bar{S})}:=\left\{k \in K: s^{k} \in S, t^{k} \in \bar{S}\right\}$ and $d_{(S, \bar{S})}:=\sum_{k \in K_{(S, \bar{S})}} d^{k}$. An appropriate aggregation of constraints (9), (10), and nonnegativity of the variables results in the following base 
cutset inequality:

$$
\sum_{u v \in(S, \bar{S})} \sum_{w=1}^{W_{u v}} \sum_{m=1}^{M_{u v}^{w}} b_{u v}^{w m} y_{u v}^{w m} \geq d_{(S, \bar{S})}
$$

In the sequel, we introduce strong inequalities obtained by Chvátal-Gomory (CG) rounding of cutset inequalities.

Type 1 Given a cutset $(S, \bar{S})$, let

$$
a_{u v}:=\min _{w=1, \ldots, W_{u v}} \min _{m=1, \ldots, M_{u v}^{w}} b_{u v}^{w m}
$$

for $u v \in(S, \bar{S})$. By 12 and $\left.a_{(S, \bar{S})}:=\sum_{u v \in(S, \bar{S})} a_{u v}, 16\right)$ can be equivalently formulated as

$$
\sum_{u v \in(S, \bar{S})} \sum_{w=1}^{W_{u v}} \sum_{m=1}^{M_{u v}^{w}}\left(b_{u v}^{w m}-a_{u v}\right) y_{u v}^{w m} \geq d_{(S, \bar{S})}-a_{(S, \bar{S})}
$$

Now, let $a$ be the maximal coefficient $\left(b_{u v}^{w m}-a_{u v}\right)$ at the left hand side of inequality (17). By CG rounding, we obtain the valid inequality Type 1 as follows:

$$
\sum_{u v \in(S, \bar{S})} \sum_{w=1}^{W_{u v}} \sum_{m=1}^{M_{u v}^{w}} 1_{u v}^{w m} y_{u v}^{w m} \geq\left\lceil\frac{d_{(S, \bar{S})}-a_{(S, \bar{S})}}{a}\right\rceil
$$

where $1_{u v}^{w m}=1$ if $b_{u v}^{w m}>a_{u v}$, and 0 otherwise.

In general, the LP relaxation of $(8)-(10),(12)-(13),(15)$ does not satisfy $(18)$ although all integer solutions have to satisfy it. Hence, the inequality is valid and can enhance the solving of the ILP. Under certain conditions, (18) defines a facet of the convex hull of feasible solutions (cf. [10]).

Type 2 Given a cutset $(S, \bar{S})$, let $a_{u v}^{\prime}$ be the second smallest capacity coefficient $b_{u v}^{w m}$ of inequality 16 for $u v \in(S, \bar{S})$, and $a^{\prime}:=\max _{u v \in(S, \bar{S})} a_{u v}^{\prime}$. We can apply CG rounding directly to (16):

$$
\sum_{u v \in(S, S)} \sum_{w=1}^{W_{u v}} \sum_{m=1}^{M_{u v}^{w}}\left\lceil\frac{b_{u v}^{w m}}{a^{\prime}}\right\rceil y_{u v}^{w m} \geq\left\lceil\frac{d_{(S, \bar{S})}}{a^{\prime}}\right\rceil
$$

Moreover, from the sum of constraints $(12)$ associated with the cutset $(S, \bar{S})$, we have

$$
\sum_{u v \in(S, \bar{S})} \sum_{w=1}^{W_{u v}} \sum_{m=1}^{M_{u v}^{w}} y_{u v}^{w m}=|(S, \bar{S})|
$$

and subtracting (20) from (19), we obtain the valid inequality Type 2 as follows:

$$
\sum_{u v \in(S, \bar{S})} \sum_{w=1}^{W_{u v}} \sum_{m=1}^{M_{u v}^{w}}\left(\left\lceil\frac{b_{u v}^{w m}}{a^{\prime}}\right\rceil-1\right) y_{u v}^{w m} \geq\left\lceil\frac{d_{(S, \bar{S})}}{a^{\prime}}\right\rceil-|(S, \bar{S})|
$$

At least two coefficients $\left(\left\lceil\frac{b_{w v}^{w w}}{a^{\prime}}\right\rceil-1\right)$ for every arc $u v \in(S, \bar{S})$ are equal to 0 , and the cutset inequality is indeed different from (18). Again, it can be shown that (21) defines a facet of the convex hull of feasible solutions under certain conditions (beyond the scope of this paper). 


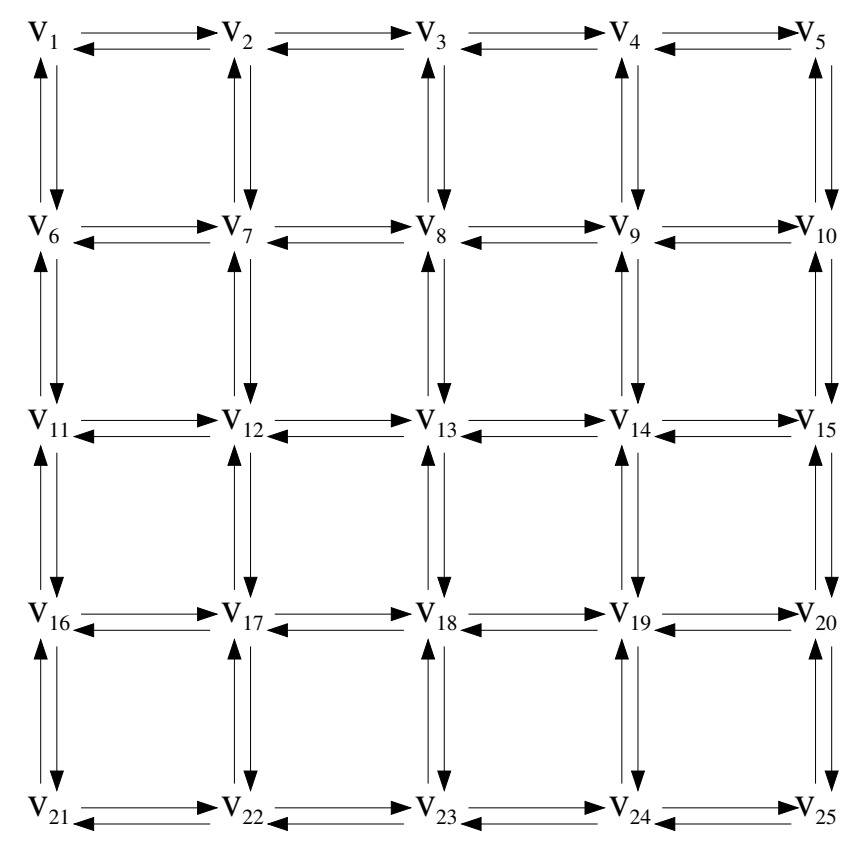

Figure 1: $5 \times 5$ grid instance

\section{Computational results}

We have performed preliminary computational experiments on a $5 \times 5$ grid instance (with 25 RBSs, 80 directional links, and 50 demands) (available at http://www.di.unipi.it/optimize/Data/MMCF.html) which originates from [6]. We consider two bandwidth choices for every link: $7 \mathrm{MHz}(28 \mathrm{MHz})$ with costs of $\$ 1,000(\$ 6,000)$. We assume that links operating at $7 \mathrm{MHz}(28 \mathrm{MHz})$ are designed to use 128-QAM (256-QAM), with availability of 99.9\%. In fading conditions, these links will use 16-QAM (32-QAM). We do not consider error correction codes, therefore bandwidth efficiencies are as presented in Table 1. We employ the ILP counterpart of the joint chance-constrained formulation since, in general, it is more appropriate to cope with practical instances of this problem. Computations were carried out on a Linux machine with $3.20 \mathrm{GHz}$ Intel Xeon W5580 CPU (8 Threads) and 64 GB RAM, using IBM ILOG CPLEX 12.1 as underlying solver.

Price of reliability Since we assume the same availability for every link (independent of the bandwidth choice) and under the hypothesis that the modulation schemes employed in fading conditions can guarantee an availability of $100 \%$, instead of explicitly setting the infeasibility tolerance $\varepsilon$, we can specify the maximum number of links $N$ that we suppose to use the highest modulation scheme. To prove that, let us rewrite (15) considering these assumptions $\left(\rho_{u v}^{w 1}=1\right.$, availability for lowest modulation schemes, and $\rho_{u v}^{w 2}=\rho$, availability for highest modulation schemes):

$$
\sum_{u v \in E} \sum_{w=1}^{W_{u v}}\left(\log (1) y_{u v}^{w 1}+\log (\rho) y_{u v}^{w 2}\right) \geq \log (1-\varepsilon)
$$




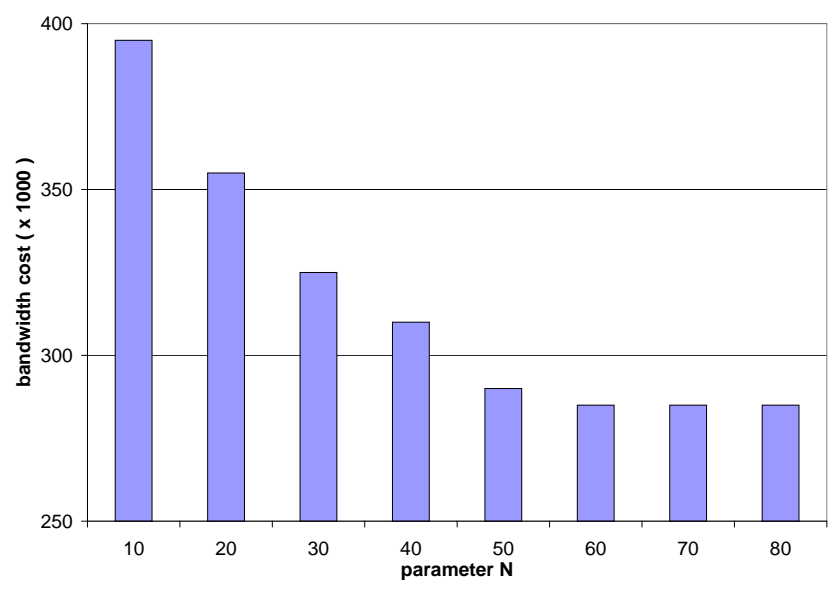

Figure 2: Bandwidth cost as a function of the infeasibility tolerance

Thus, in this scenario, (15) can be replaced by:

$$
\sum_{u v \in E} \sum_{w=1}^{W_{u v}} y_{u v}^{w 2} \leq\left\lfloor\frac{\log (1-\varepsilon)}{\log (\rho)}\right\rfloor=: N
$$

Note that a larger infeasibility tolerance $\varepsilon$ implies a larger value $N$. To observe the evolution of the bandwidth cost as a function of the infeasibility tolerance, we ran tests for $N=0,10, \ldots, 80$. The solutions (see Fig. 2), were obtained by solving the (enhanced) formulation D (as further described in Table 2) to optimality, which took several hours (even days) of computation for each instance. As illustrated in Fig. 2, the total bandwidth cost decreases as we admit larger values for the infeasibility tolerance ( $N$ augments). For $N=0$, assuming the lowest modulation schemes for all links, the network cannot provide enough capacity to satisfy all the traffic demands, hence this problem is infeasible. For $N=10(\varepsilon=0.01)$, the bandwidth cost is $38.6 \%$ higher than the bandwidth cost for $N=80(\varepsilon=0.077)$ and $68.4 \%$ higher compared to the case where we do not use any optimization (i.e., we assign $28 \mathrm{MHz}$ for every link). For $N=10,20, \ldots, 50$, the decrease in costs becomes evident with the degradation of the network reliability. For $N=60,70,80$, the reliability constraint (23) does not affect the cost of the solutions because the number of links we need to consider using the highest modulation schemes to satisfy all the traffic requirements is smaller than 60 .

Comparison of the formulations To study the gain of applying the valid inequalities introduced in Section 4, we performed tests for each of the four different formulations A, B, C, D, according to Table 2. Note that in formulations B and D also inequalities (16) are added although these do not contribute to an improvement of the objective - the ILP solver, however, can benefit from those to generate its own valid inequalities. We manually identified a restricted, but sufficiently large (432 cutsets in total), set of cutsets of the type $(S, \bar{S})$ that were used to generate the valid inequalities. As typical examples, according to the node labeling of Fig. 1, we considered the following sets $S$ :

$$
\left\{v_{1}, v_{2}, v_{3}, v_{4}, v_{5}, v_{6}, v_{7}, v_{8}, v_{9}, v_{10}\right\}
$$




\begin{tabular}{llll}
\hline Formulation & Cutset & Type1 & Type2 \\
\hline A & no & no & no \\
B & yes & no & no \\
C & no & yes & yes \\
D & yes & yes & yes \\
\hline
\end{tabular}

Table 2: Different formulations w.r.t the valid inequalities

$$
\begin{gathered}
\left\{v_{4}, v_{5}, v_{9}, v_{10}, v_{14}, v_{15}, v_{19}, v_{20}, v_{24}, v_{25}\right\}, \\
\left\{v_{1}, v_{2}, v_{3}, v_{6}, v_{7}, v_{11}\right\}, \\
\left\{v_{1}, v_{2}, v_{6}, v_{7}, v_{11}, v_{12}\right\}, \\
\left\{v_{18}, v_{19}, v_{20}, v_{21}, v_{22}, v_{23}, v_{24}, v_{25}\right\} .
\end{gathered}
$$

Due to computational limitations, first a limit of 30000 nodes (LP relaxations) of the branchand-bound process is set. In addition, as the size and the complexity of the LP relaxations vary according to each formulation, instead of imposing a limit on the number of nodes, we also perform tests where we set a time limit of 1 hour of computation. All other solver settings are preserved at their defaults.

Fig. 3 illustrates the optimality gaps achieved for the different formulations considering a limit on the number of nodes (Fig. 3(a)) and execution time (Fig. 3(b)), along with the best feasible solutions (Fig. 3(c) and lower bound values (Fig. 3(d) considering the first scenario. With respect to the optimality gap, the adding of valid inequalities improves the performance of the ILP solver in both scenarios. Formulation B performs significantly better than the basic problem formulation A. Formulation $\mathrm{C}$ also improves the basic problem formulation $\mathrm{A}$ for most cases, but it does not perform as well as formulation B. Actually, the valid inequalities Type 1 and Type 2 are more useful in conjunction with cutset inequalities. In fact, formulation $\mathrm{D}$ presents the best results in terms of the achieved optimality gap for most cases. Nevertheless, there are no significant differences among feasible solutions (see Fig. 3(c) - lower values mean better solutions) found by each formulation to explain the better performance of formulations B and D. In Fig. 4, we compare the performance of the different formulations according to the number of LP relaxations. Note that, in general, the optimality gap decreases rather quickly until finding a barrier in a given level, while the lower bounds defined by the LP relaxations improve rather slowly, suggesting that much computational effort is made to prove the optimality of the current feasible solutions. Formulations B and D coped better with the task of finding tighter lower bounds (see Fig. $3(\mathrm{~d})$ - higher values mean better lower bounds), and this explains in part why these formulations provide lower optimality gaps.

\section{Conclusion}

In this paper, we have presented a chance-constrained programming approach to tackle the problem of assigning bandwidth for reliable fixed broadband wireless networks. We introduced mathematical formulations and proposed cutset-based valid inequalities for this problem. In our computational studies, we discussed the price of reliability and compared the performance of different cutset inequalities. 


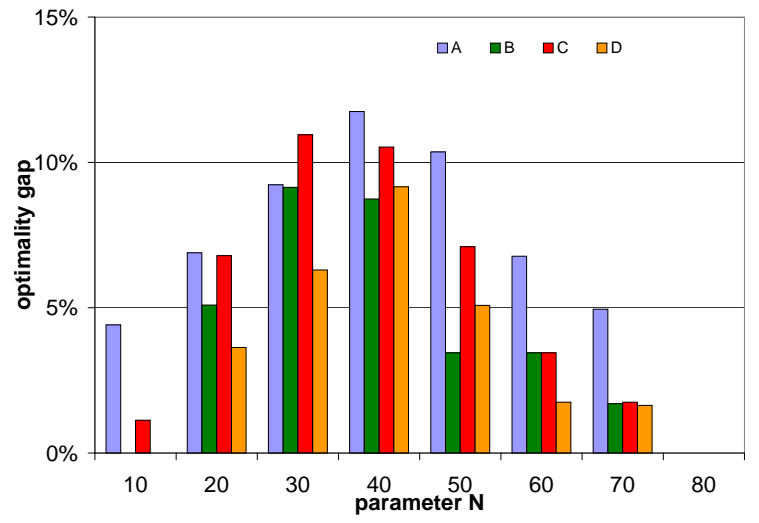

(a) Gap within nodes limit

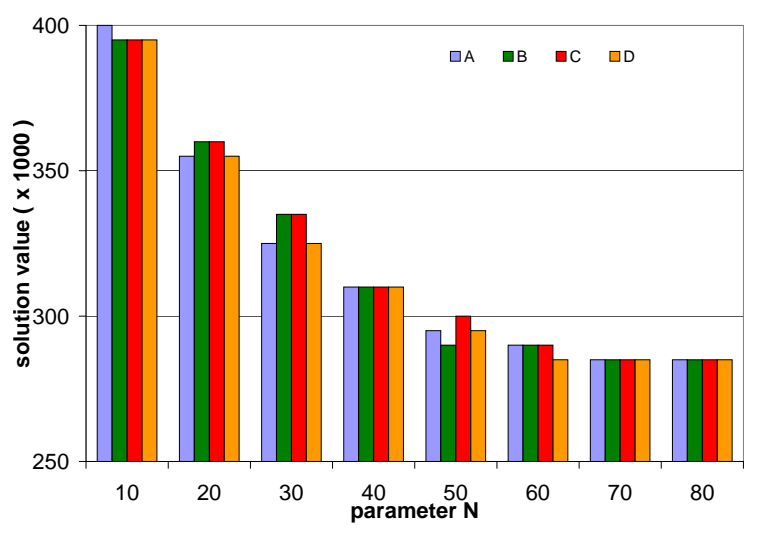

(c) Best solution

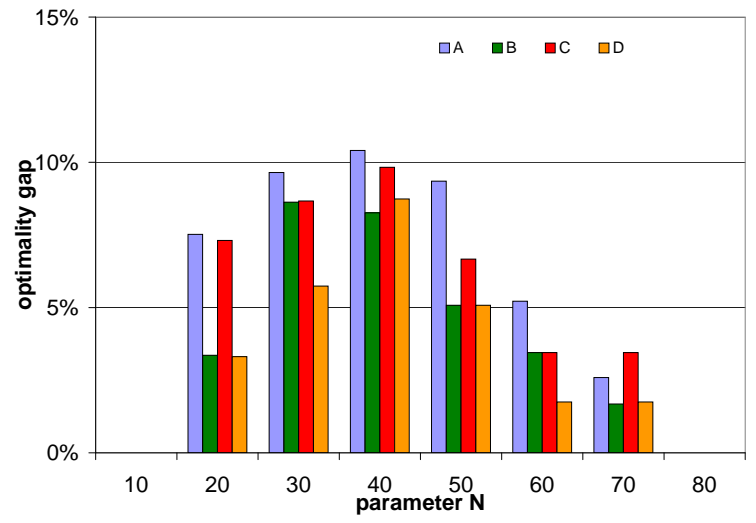

(b) Gap within time limit

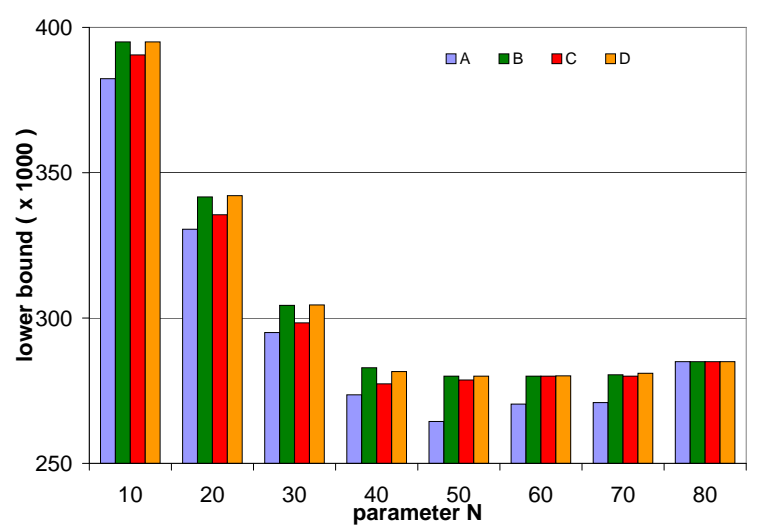

(d) Lower bound

Figure 3: Optimality gap, best solution and lower bound values achieved for the different formulations

As future work, we intend to investigate more realistic network topologies and radio scenarios. In addition, we envisage to study the impact of different aspects (e.g., equipment and frequency diversity, traffic fluctuations) on the reliability of the network.

\section{Acknowledgment}

This work has been supported by ANR DIMAGREEN and ECOSCELLS, the PATHFINDER project, Région PACA, and SME 3ROAM as well as the excellence initiative of the German federal and state governments and by the UMIC Research Centre at RWTH Aachen University. Financial support by the Villum Kann Rasmussen foundation is greatfully acknowledged.

\section{References}

[1] Harry Anderson. Fixed Broadband Wireless System Design. John Wiley \& Sons, New Jersey, first edition, 2003. 
[2] D. Coudert, N. Nepomuceno, and H. Rivano. Power-efficient radio configuration in fixed broadband wireless networks. Comput. Commun., 33(8):898-906, 2010.

[3] R. K. Crane. Electromagnetic wave propagation through rain. John Wiley \& Sons, 1996.

[4] Andrea Goldsmith and S. Chua. Variable-rate variable-power MQAM for fading channels. IEEE Trans. Commun, 45(10):1218-1230, 1997.

[5] Olivier Klopfenstein. Solving chance-constrained combinatorial problems to optimality. Comput. Optim. Appl., 45(3):607-638, 2010.

[6] Torbjörn Larsson and Di Yuan. An augmented lagrangian algorithm for large scale multicommodity routing. Comput. Optim. Appl., 27(2):187-215, 2004.

[7] H. Lehpamer. Microwave transmission networks: planning, design, and deployment. McGrawHill, 2010.

[8] James Luedtke, Shabbir Ahmed, and George L. Nemhauser. An integer programming approach for linear programs with probabilistic constraints. Math. Program., 122(2):247-272, 2010.

[9] A. Prékopa. Stochastic Programming. Kluwer, 1995.

[10] C. Raack, A. M. C. A. Koster, S. Orlowski, and R. Wessäly. On cut-based inequalities for capacitated network design polyhedra. Networks, online, 2010.

[11] A. Shapiro, D. Dentcheva, and A. Ruszczynski. Lectures on Stochastic Programming: Modeling and Theory. SIAM, 2009.

[12] A. Vigants. Space-diversity engineering. Bell System Tech. J., 54(1):103-142, 1975. 


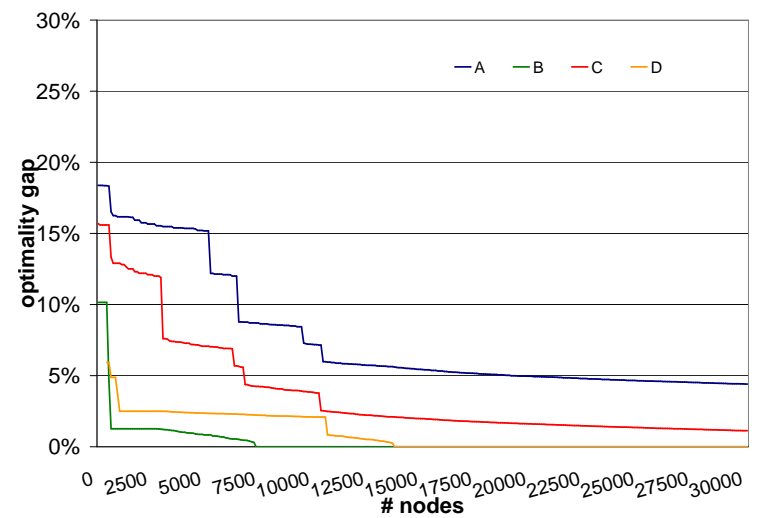

(a) $\mathrm{N}=10$

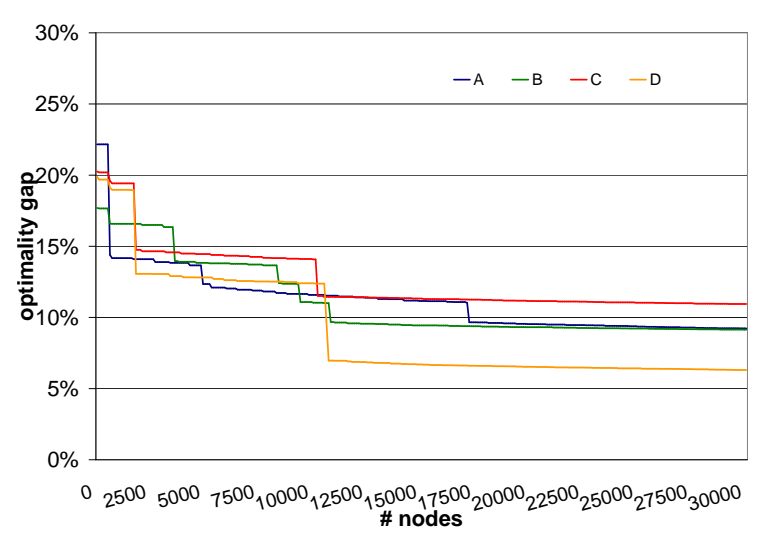

(c) $\mathrm{N}=30$

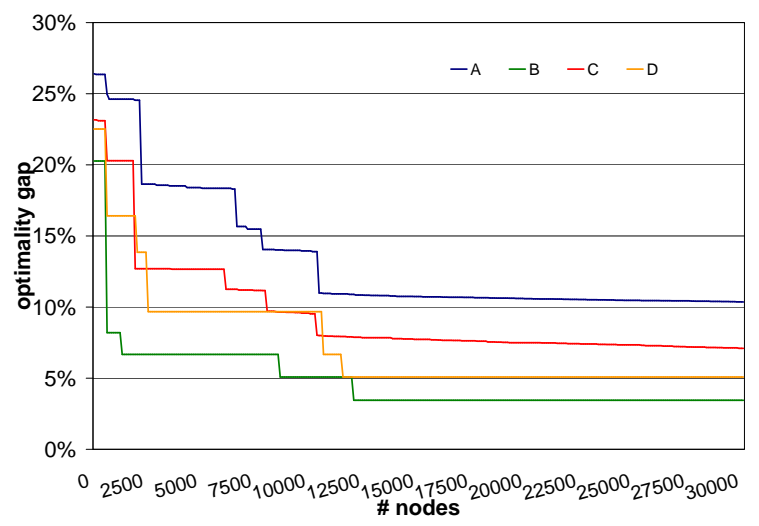

(e) $\mathrm{N}=50$

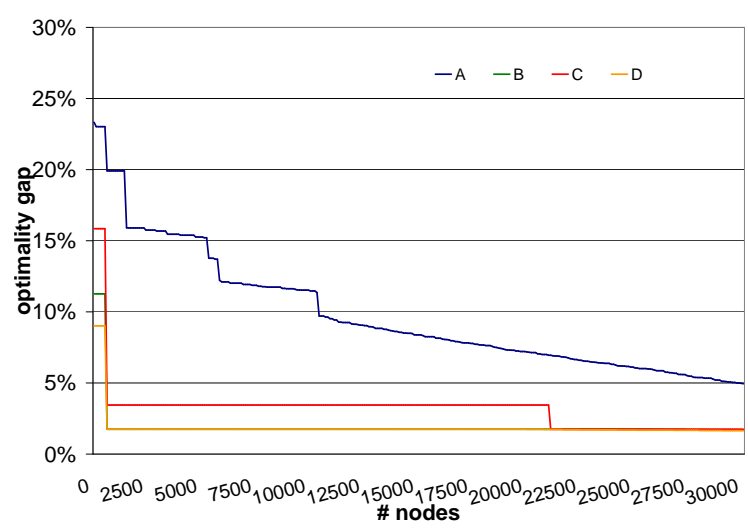

(g) $\mathrm{N}=70$

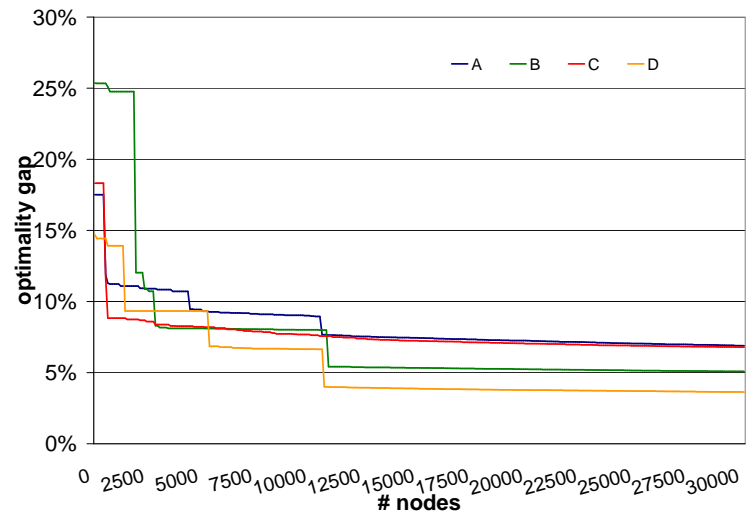

(b) $\mathrm{N}=20$

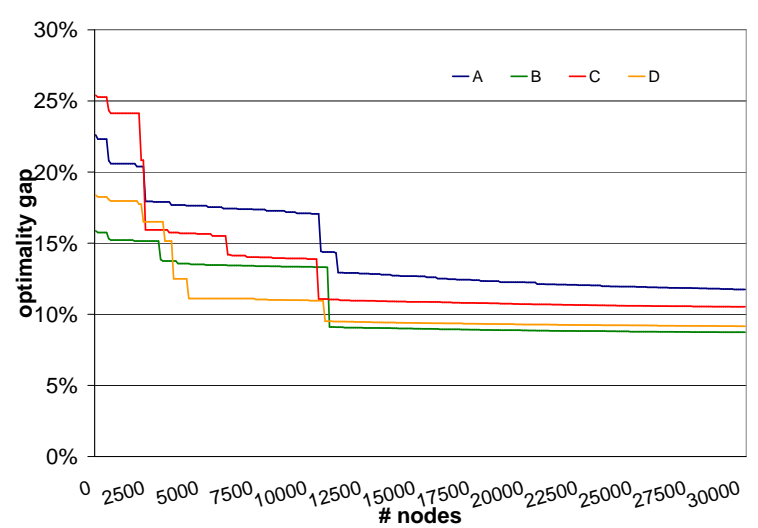

(d) $\mathrm{N}=40$

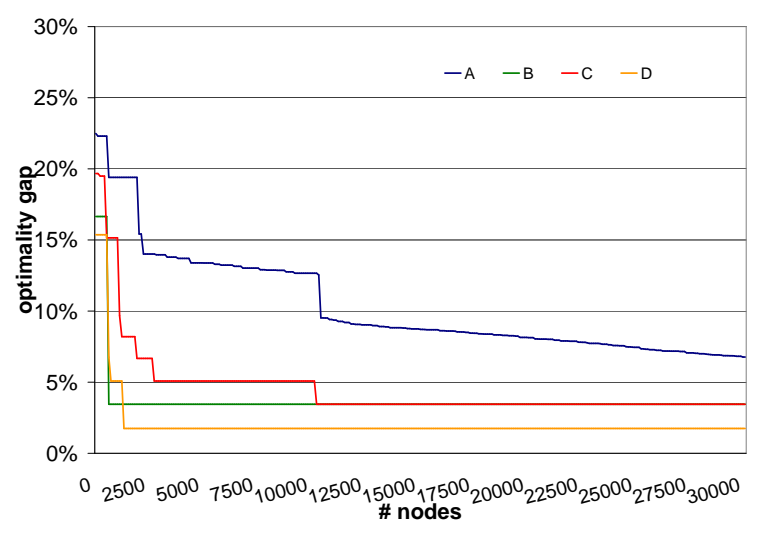

(f) $\mathrm{N}=60$

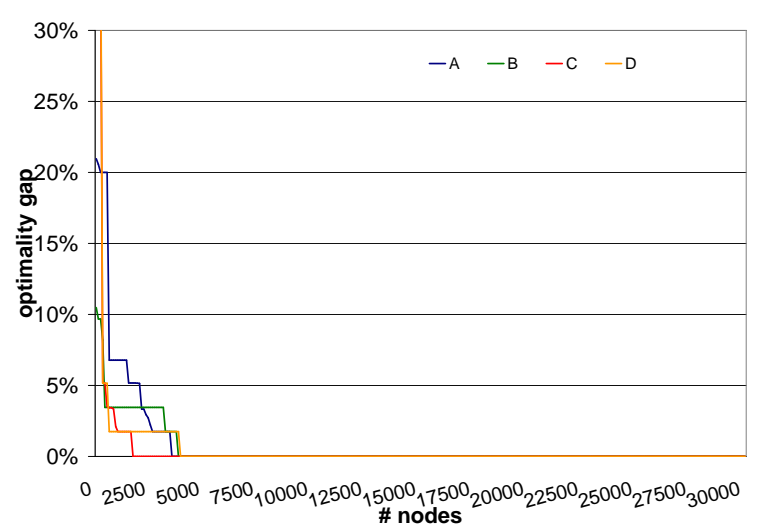

(h) $\mathrm{N}=80$

Figure 4: Performance of the different formulations according to the number of LP relaxations 